\title{
Increased SHP-1 expression results in radioresistance, inhibition of cellular senescence, and cell cycle redistribution in nasopharyngeal carcinoma cells
}

\author{
Ziyi Sun $^{1+}$, Xiaofen Pan ${ }^{1,2+}$, Zhenwei Zou', Qian Ding ${ }^{1}$, Gang Wu ${ }^{1}$ and Gang Peng ${ }^{1 *}$
}

\begin{abstract}
Background: Radioresistance is the main limit to the efficacy of radiotherapy in nasopharyngeal carcinoma (NPC). SHP-1 is involved in cancer progression, but its role in radioresistance and senescence of NPC is not well understood. This study aimed to assess the role of SHP-1 in the radioresistance and senescence of NPC cells.

Methods: SHP-1 was knocked-down and overexpressed in CNE-1 and CNE-2 cells using lentiviruses. Cells were irradiated to observe their radiosensitivity by colony forming assay. BrdU incorporation assay and flow cytometry were used to monitor cell cycle. A $\beta$-galactosidase assay was used to assess senescence. Western blot was used to assess SHP-1, p21, p53, pRb, Rb, H3K9Me3, HP1y, CDK4, cyclin D1, cyclin E, and p16 protein expressions.

Results: Compared with CNE-1-scramble shRNA cells, SHP-1 downregulation resulted in increased senescence $(+107 \%, P<0.001)$, increased radiosensitivity, higher proportion of cells in G0/G1 $(+33 \%, P<0.001)$, decreased expressions of CDK4 ( $-44 \%, P<0.001)$, cyclin D1 ( $-41 \%, P=0.001)$, cyclin $\mathrm{E}(-97 \%, P<0.001)$, Rb $(-79 \%, P<0.001)$, and pRb $(-76 \%, P=0.001)$, and increased expression of p16 $(+120 \%, P=0.02)$. Furthermore, SHP-1 overexpression resulted in radioresistance, inhibition of cellular senescence, and cell cycle arrest in the S phase. Levels of p53 and p21 were unchanged in both cell lines (all $P>0.05$ ).
\end{abstract}

Conclusion: SHP-1 has a critical role in radioresistance, cell cycle progression, and senescence of NPC cells. Down-regulating SHP-1 may be a promising therapeutic approach for treating patients with NPC.

Keywords: Nasopharyngeal carcinoma, SHP-1, Cellular senescence, Cell cycle distribution, p16, Rb

\section{Background}

Nasopharyngeal carcinoma (NPC) is a cancer with a distinctly skewed geographic and ethnic distribution, and is endemic in Southern China and South East Asia [1]. Indeed, type I NPC represents $25 \%$ of the cases in North America and $2 \%$ in Southern China, while type III represents $95 \%$ of the cases in China and $63 \%$ in North America [1]. NPC mostly affects men (men:women ratio of 4.4:1), and the median age at diagnosis is in the early $60 \mathrm{~s}$. Worldwide incidence is $<1$ per 100,000 for either men or women, but the incidence is $20-30$ per 100,000 in Hong

\footnotetext{
* Correspondence: penggangsci@sina.com

'Equal contributors

${ }^{1}$ Cancer Center, Union hosipital, Wuhan 430022, Hubei Province, China

Full list of author information is available at the end of the article
}

Kong and 15-20 per 100,000 in Guangdong [1]. Tobacco and alcohol are the two main risk factors for NPC [2-4]. Human papillomavirus and Epstein-Barr virus also increase the risk of NPC, particularly in endemic regions $[5,6]$. Mean survival of patients with stage I, II, or III NPC is about 3 years [7].

Radiotherapy (RT) is the main treatment for NPC since radical resection is typically not possible [4]. The advent of megavoltage radiotherapy has transformed a once lethal cancer into one that is readily curable. Non-keratinizing undifferentiated carcinoma (type III NPC) is the most common of the three forms of NPC, and is sensitive to radiations [4]. Overall survival exceeding $50 \%$ at five years may now be achieved $[8,9]$. 
However, radioresistance is the main factor limiting the benefits from radiotherapy [10]. The mechanisms of radioresistance are mostly unknown. Recent studies using microarrays have explored the genes involved in the radioresistance of solid tumors such as cervix, pancreas, mouth, lung, and esophagus and failed to identify common genes [11]. Nevertheless, recent studies have shown that a number of proteins involved in the cell cycle (gp96, GDF15, PTEN) were involved in the radioresistance of NPC $[11,12]$.

SHP-1 (initially designated as SHPTP-1, SHP, HCP, and PTPIC) is a cytosolic protein tyrosine phosphatase expressed primarily in hematopoietic cells [13]. SHP-1 has been proposed as a candidate tumor suppressor gene in lymphoma and solid cancers [14]. SHP-1 can play either negative or positive roles in regulating signal transduction pathways and is differentially expressed in a number of cancer cell lines [14-16]. Therefore, SHP-1 appears to have different roles and mechanisms in the regulation of cell cycle and cell proliferation in different types of tumors.

Recent data validated the early idea that cellular senescence is important for tumor suppression $[17,18]$. Cellular senescence is a barrier to tumorigenesis and contributes to mammalian aging [19]. Furthermore, cellular senescence depends critically on two powerful tumor suppressor pathways: the p53 and pRb/p16INK4a pathways. These pathways are known to regulate cellular senescence/immortalization including the p16INK4a/pRB, p19ARF/p53/ p21CIP1/WAF1, and PTEN/p27KIP1 pathways [20-22].

A previous study has shown that SHP-1 knockdown resulted in a G1/S arrest and an increase in the expression of p16 [23]. This previous study investigated the association between SHP-1 and p16, since p16 has previously been demonstrated to be silenced in the vast majority of NPCs [24], which suggested that SHP-1 may regulate cellular senescence through the p16 pathway.

Therefore, the aim of the present study was to study the association between expression of SHP-1 and cellular senescence, radioresistance and cell cycle distribution in NPC cells. This study is the first to propose that SHP-1 regulates a senescence response.

\section{Methods}

\section{Cell culture}

The human NPC cell lines CNE-1 and CNE-2 were obtained from the Cell Bank of Sun Yat-sen University (Guangzhou, China), and cultured in RPMI 1640 (Invitrogen Inc., Carlsbad, CA, USA) supplemented with $10 \%$ fetal bovine serum (FBS, Invitrogen Inc., Carlsbad, CA, USA) and $1 \%$ penicillin/streptomycin (Invitrogen Inc., Carlsbad, CA, USA). Cells were kept at $37{ }^{\circ} \mathrm{C}$ in $5 \% \mathrm{CO}_{2}$ atmosphere. The CNE-1 cell line was established in 1978 from a patient with NPC [25], and the CNE-2 cell line was established in 1983 from a poorly differentiated NPC [26]. The CNE-2 cell line has been shown to be less radio-resistant than CNE-1 [27], and the DNA repair mechanisms seem to be more efficient in the CNE-1 cell line $[27,28]$.

\section{SHP-1 overexpression and knockdown mediated by lentiviruses}

For SHP-1 knockdown, cells were plated in 24-well plates and cultured with $0.5 \mathrm{ml}$ of RPMI 1640 supplemented with $5 \%$ FBS and $1 \%$ penicillin/streptomycin for $24 \mathrm{~h}$. Cells were then transduced with $50 \mu \mathrm{l}\left(8.6 \times 10^{9}\right.$ copies $\left./ \mathrm{ml}\right)$ of lentivirus-mediated SHP-1-shRNA vector (lot: LPHSH015860-LVRH1MP) and scramble shRNA vector (lot: LP-CSHCTR001-LVRH1MP) (GeneCopoeia, Guangzhou, China) for $48 \mathrm{~h}$. These vectors contained a puromycin resistance gene for the selection of transduced cells. Then, the transduced cells were digested with trypsin, plated in 6-well plates, and cultured in RPMI 1640 supplemented with $10 \%$ FBS, $1 \%$ penicillin/streptomycin and $2 \mu \mathrm{g} / \mathrm{ml}$ of puromycin for 12 days to screen for stably transduced cells. The medium containing puromycin was changed every three days. Puromycin-resistant clones were selected. SHP-1 mRNA and protein expressions were determined by real-time RT-PCR and western blot.

For SHP-1 overexpression, cells were plated in 24-well plates and cultured with $0.5 \mathrm{ml}$ of RPMI 1640 supplemented with $15 \%$ FBS and $1 \%$ penicillin/streptomycin for $24 \mathrm{~h}$. Cells were then transduced with $50 \mu \mathrm{l}\left(8.6 \times 10^{9}\right.$ cop$\mathrm{ies} / \mathrm{ml}$ ) of lentivirus-mediated SHP-1-overexpression vector (lot: LP-H1802-Lv201-C0010) and scramble shRNA vector (lot: LP-NEG-Lv201-0200) (GeneCopoeia, Guangzhou, China) for $48 \mathrm{~h}$. These vectors contained a puromycin resistance gene for the selection of transduced cells. Then, the transduced cells were observed using a Zeiss Axioplan 2 fluorescence microscope (Carl Zeiss $\mathrm{GmbH}$, Oberkochen, Germany) equipped with a Plan Neofluar 20x/0.5 objective, color camera Infinity X and Deltapix software (DeltaPix, Nibe, Denmark). Transduced cells were digested with trypsin, plated in 6-well plates, and cultured in RPMI 1640 supplemented with $15 \%$ FBS, $1 \%$ penicillin streptomycin and $2 \mu \mathrm{g} / \mathrm{ml}$ of puromycin for 12 days to screen for stably transduced cells. The medium containing puromycin was changed every three days. Puromycin-resistant clones were selected. SHP-1 mRNA and protein expressions were determined by real-time RT-PCR and western blot.

\section{Colony forming assay}

Cells were seeded in 6-well culture plates at different cell densities (200, 300, 600, 1500, and 4000 cells/well) and irradiated the next day using different doses $(0,2,4,6$, and $8 \mathrm{~Gy})$. The plates were incubated for 14 days, fixed with methanol, and stained with Giemsa (Sigma, St Louis, MI, USA). Colonies containing at least 50 cells were counted as a clone. A multi-target single-hit model 
was used to describe the survival fraction using the equation $\mathrm{SF}=1-\left(1-\mathrm{e}^{-\mathrm{D} / \mathrm{D} 0}\right)^{\mathrm{N}}$, where $\mathrm{SF}$ is the cell survival fraction, $\mathrm{D}$ is the radiation dose, e is the natural logarithm, D0 is the mean lethal dose, and N is the extrapolated number. Survival curves were made using these SF.

\section{BrdU incorporation assay}

Cells were cultured on glass slides and were incubated with $10 \mu \mathrm{M}$ BrdU (Sigma, St Louis, MI, USA) for $6 \mathrm{~h}$ before fixation with $4 \%$ formaldehyde. After DNA denaturation in $2 \mathrm{M} \mathrm{HCl}$ for $30 \mathrm{~min}$, cells were washed in PBS and incubated with mouse anti-human primary antibody against BrdU (RPN20AB, 1:300, AP-Biotech S.R.L., Buenos Aires, Argentica). The secondary antibody Alexa Fluor 568 goat anti-mouse (\#A110-31, 1:500, Invitrogen Inc., Carlsbad, CA, USA) was then applied for $60 \mathrm{~min}$ at room temperature, followed by a final wash in PBS. Glass slides were mounted in Vectashield Mounting Medium with HOCHEST (Vector Laboratories, Burlingame, CA, USA). Images were acquired using an AxioObserver fluorescence microscope (Carl Zeiss $\mathrm{GmbH}$, Oberkochen, Germany) equipped with a Plan Apochromat 20x/0.8 objective, camera Coolsnap HQ (Photometrics, Tucson, AZ, USA) and the Metamorph software (Universal Imaging, Bedford Hills, NY, USA). Two independent observers blinded to grouping counted the BrdU-positive cells in 35 random fields (approximately 500 cells) for each glass slide, and the percentage of BrdU-positive cells was calculated. Data analysis was performed using ImageJ $1.43 \mathrm{u}$ (National Institutes of Health, Bethesda, MD, USA).

\section{Senescence-associated $\beta$-galactosidase}

Staining for senescence-associated- $\beta$-galactosidase activity was performed using a Senescence $\beta$-Galactosidase Staining Kit (\#9860, Cell Signaling, Danvers, MA, USA) according to the manufacturer's protocol. $\beta$-galactosidase positive cells (green) were viewed by light microscopy.

\section{Immunofluorescence}

For immunofluorescence, cells were cultured on glass slides, fixed in $4 \%$ formaldehyde and permeabilized by $0.1 \%$ Triton X-100 in two consecutive steps, each for $15 \mathrm{~min}$ at room temperature. After washing with PBS, cells were blocked for $30 \mathrm{~min}$ in $10 \%$ fetal calf serum. The following primary antibodies were used: rabbit antihuman polyclonal antibody against histone $\mathrm{H} 3$ trimethylated at lysine 9 (H3K9Me3, \#07-442, 1:1,000, Millipore corp., Billerica, MA, USA) and mouse anti-human polyclonal antibody against heterochromatin protein- $1 \gamma$ (HP1 $\gamma$, \#MAB3450, 1:4,000, Millipore corp., Billerica, MA, USA). Incubation with the primary antibodies was performed for $60 \mathrm{~min}$ at room temperature and the cells were washed with PBS. The secondary antibodies Alexa
Fluor 568 goat anti-mouse (\#A110-31, 1:500, Invitrogen Inc., Carlsbad, CA, USA) and Alexa Fluor 488 goat antirabbit (\#A-11008, 1:500, Invitrogen Inc., Carlsbad, CA, USA) were then applied for $60 \mathrm{~min}$ at room temperature, followed by a final wash in PBS. Glass slides were mounted in Vectashield Mounting Medium with HOCHEST (Vector Laboratories, Burlingame, CA, USA). Confocal images were acquired using a LSM-510 microscope (Carl Zeiss $\mathrm{GmbH}$, Oberkochen, Germany) equipped with a PlanApochromat 63x/1.4 oil immersion objective and the ZEN2009 software (Carl Zeiss GmbH, Oberkochen, Germany). Identical image acquisition parameters were used for quantitative and comparative imaging. Percentage of senescence-associated heterochromatin foci (SAHF)positive cells (based on Hoechst staining) and numbers of H3K9Me3 and HP1Y foci per cell were counted by two independent observers blinded to grouping from at least 200 cells from each glass slide. Data analysis was performed using Image $1.43 \mathrm{u}$ (National Institutes of Health, Bethesda, MD, USA).

\section{Flow cytometry}

After transduction with the indicated lentiviruses, cells were fixed overnight with $70 \%$ ethanol, followed by resuspension in PBS containing $1 \mathrm{mg} / \mathrm{ml}$ of RNase and $50 \mu \mathrm{g} / \mathrm{ml}$ of propidium iodide (Sigma, St Louis, MI, USA). Cellular DNA content was determined using a FACScan flow cytometer (BD Biosciences, Franklin Lake, NJ, USA).

\section{Real-time RT-PCR}

Total RNA was extracted using TRIzol (Invitrogen, NM, USA), and cDNA synthesis was performed using the Prime Script RT-PCR kit (Takara, Shiga, Japan), according to the manufacturers' instructions. Then, real-time PCR was performed using SYBR Green PCR Master Mix (Applied Biosystems, USA) in a PCR amplifier (ABI Prism 7000 , USA). The StepOne ${ }^{\mathrm{TM}}$ Software v2.1 was used to analyze the data. The primer sequences for SHP-1 were: Forward, 5'-ACCATCATCCACCTCAAGTACC-3' and Reverse, 5'-CTGAGCACAGAAAGCACGAA-3'. $\beta$-actin was used as an internal control, and the primer sequences were: Forward, 5'-GATGAGATTGGCATGGCTTT-3' and Reverse, 5'-CACCTTCACCGTTCCAGTTT-3'. $2^{-\Delta \Delta C t}$ was calculated to represent the relative mRNA expression of target genes.

\section{Western blot}

Cells were lysed with the RIPA buffer (Invitrogen Inc., Carlsbad, CA, USA), and then centrifuged $(12,000 \mathrm{rpm}$, $15 \mathrm{~min}, 4^{\circ} \mathrm{C}$ ). Protein content was measured using a BCA assay. Equal amounts of proteins $(20-80 \mu \mathrm{g})$ were separated with $10 \%$ sodium docecyl sulfate-polyacrylamide gel electrophoresis, and transferred to polyvinylidene difluoride 
membranes (Santa Cruz Biotechnology, Santa Cruz, CA, USA). Membranes were blocked with $5 \%$ BSA for $1 \mathrm{~h}$, and probed using mouse anti-human p21 (1:400; Abcam, Cambridge, MA, USA), mouse anti-human p53 (1:1000, Cell Signaling, Danvers, MA, USA), mouse anti-human pRb (Ser795) (1:1000, Cell Signaling, Danvers, MA, USA), mouse anti-human Rb (1:2000, Cell Signaling, Danvers, MA, USA), mouse anti-human H3K9Me3 (1:500, Millipore corp., Billerica, MA, USA), mouse anti-humanHP1- $\gamma$ (1:500, Millipore corp., Billerica, MA, USA), mouse antihuman SHP1 (1:1000, Epitomic, San Francisco, CA, USA), mouse anti-human CDK4 (1:1000, Abcam, Cambridge, MA, USA), mouse anti-human cyclin D1 (1:1000, Epitomic, San Francisco, CA, USA), mouse anti-human cyclin E (1:1000, Bioworld Technology Inc., Louis Park, MN, USA), mouse anti-human p16 (1:1000, Santa Cruz Biotechnology, Santa Cruz, CA, USA), or rabbit anti-human $\beta$-actin (1:2500; Sigma, St Louis, MI, USA) polyclonal antibodies overnight at $4{ }^{\circ} \mathrm{C}$. Primary antibodies were detected using horseradish peroxidase-conjugated secondary antibodies (Invitrogen Inc., Carlsbad, CA, USA), and were visualized using enhanced chemiluminescence (SuperSignal, Pierce, Rockford, IL, USA). Grayscale images were analyzed using Image $1.43 \mathrm{~b}$ (National Institutes of Health, Bethesda, MD, USA).

\section{Statistical analysis}

Statistical analysis was performed using SPSS 12.0 (SPSS Inc., Chicago, IL, USA). Data are expressed as means \pm standard deviation (SD) of at least three independent experiments and evaluated by one-way analysis of variance (ANOVA) with the least significant difference (LSD) test for post hoc analysis or student's $t$-test. $P$-values $<0.05$ were considered statistically significant.

\section{Results}

Expression of SHP-1 by lentivirus-mediated RNA interference and overexpression

Figure 1a and b show the baseline expression of SHP-1 and radiosensitivity in CNE-1 and CNE-2 cells. The majority of cells above $90 \%$ displayed green fluorescence $48 \mathrm{~h}$ after lentivirus transduction (Fig. 1c and d). Real-time RT-PCR showed that SHP-1 mRNA expression was suppressed by $62.5 \%$ in CNE-1 cells transduced with lentivirus-mediated SHP-1 shRNA (CNE-1 SHP-1 shRNA), compared with cells transduced with lentivirus-mediated scramble shRNA (CNE-1-scramble shRNA), while SHP-1 mRNA expression was overexpressed 249.2 folds in CNE-2 cells transduced with lentivirus-mediated SHP-1 overexpression (CNE-2 SHP-1 overexpression), compared with cells transduced with lentivirus vector (CNE-2-empty vector) (Fig. 1e). Realtime RT-PCR results were confirmed by western blotting (Fig. 1f, g, and h), i.e. $-47.2 \%$ in CNE-1 SHP-1 shRNA cells, and $+90.3 \%$ in CNE-2 SHP-1 overexpression cells.
Effects of SHP-1 knockdown and overexpression in CNE-1 and CNE-2 cells on radiosensitivity

Survival curves of CNE-1, CNE-1-empty vector, CNE-1scramble shRNA, CNE-1 SHP-1 shRNA, and CNE-1 SHP-1 overexpression cells after irradiation are shown in Fig. 2a, and the survival curves of CNE-2, CNE-2-empty vector, CNE-2-scramble shRNA, CNE-2 SHP-1 shRNA, and CNE-2 SHP-1 overexpression cells after irradiation are shown in Fig. 2b. The curves show that SHP-1 overexpression cells had a higher radioresistance compared with nontransduced cells or cells transduced with empty vector $(P<0.001$ for all radiation doses) (Fig. 2). Notably, SHP-1 overexpression cells had higher D0, Dq, and SF2 values compared with non-transduced cells, indicating higher radioresistance (Table 1). By contrast, D0, Dq, and SF2 values were similar between the non-transduced cells and cells transduced with empty vector. On the other hand, SHP-1 shRNA cells had a lower radioresistance compared with non-transduced cells or cells transduced with scramble shRNA $(P<0.001$ for all radiation doses) (Fig. 2 and Table 1).

\section{Effects of SHP-1 knockdown in CNE-1 cells and overexpression in CNE-2 cells on NPC cell senescence}

Cell cycle arrest is the central feature of senescent cells. Morphologically, CNE-2 and CNE-2-empty vector cells were vacuolated, flattened and much larger in size compared with CNE-2 SHP-1 overexpression cells (Fig. 3a). $\beta$-galactosidase staining revealed higher senescence in CNE-1 SHP-1 shRNA cells compared with CNE-1scramble shRNA cells $(23.6 \pm 3.4 \%$ vs. $11.4 \pm 1.8 \%, P<$ 0.001 ), and lower senescence in CNE-2 SHP-1 overexpression cells compared with CNE-2-empty vector cells (3.6 $\pm 2.7 \%$ vs. $13.2 \pm 3.3 \%, P=0.001)$ (Fig. 3a).

H3K9Me3 and HP1 $\gamma$ analyses showed that more CNE-1 SHP-1 shRNA cells were positive for H3K9Me3 (13.6 \pm $2.7 \%$ vs. $4.6 \pm 1.9 \%, P<0.001)$ and $\mathrm{HP} 1 \gamma(12.0 \pm 2.2 \%$ vs. $2.8 \pm 1.5 \%, P<0.001)$ compared with CNE-1-scramble shRNA cells, while fewer CNE-2 SHP-1 overexpression cells were positive for H3K9Me3 $(2.6 \pm 1.5 \%$ vs. $9.6 \pm$ $2.1 \%, P<0.001)$ and HP1 $\gamma(3.6 \pm 1.5 \%$ vs. $10.0 \pm 2.3 \%$, $P=0.001$ ) (Fig. 3b). These results were confirmed by western blot for $\mathrm{H} 3 \mathrm{~K} 9 \mathrm{Me} 3$ and $\mathrm{HP} 1 \gamma$, i.e. $+292 \%$ for $\mathrm{H} 3 \mathrm{~K} 9 \mathrm{Me} 3$ and $+54 \%$ for HP1 $\gamma$ in CNE-1 SHP-1 shRNA cells compared with CNE-1-scramble shRNA cells, and $-37 \%$ for $\mathrm{H} 3 \mathrm{~K} 9 \mathrm{Me} 3$ and $-83 \%$ for $\mathrm{HP} 1 \gamma$ in CNE-2 SHP-1 overexpression cells compared with CNE-2-empty vector cells (all $P<0.001$ ) (Fig. 3c).

\section{Effects of SHP-1 knockdown in CNE-1 cells and} overexpression in CNE-2 cells on NPC cell cycle distribution As shown in Fig. 4a, compared with CNE-1-scramble shRNA cells, CNE-1 SHP-1 shRNA cells had a higher proportion of cells in G0/G1 (80.0 $\pm 1.7 \%$ vs. $60.3 \pm$ 


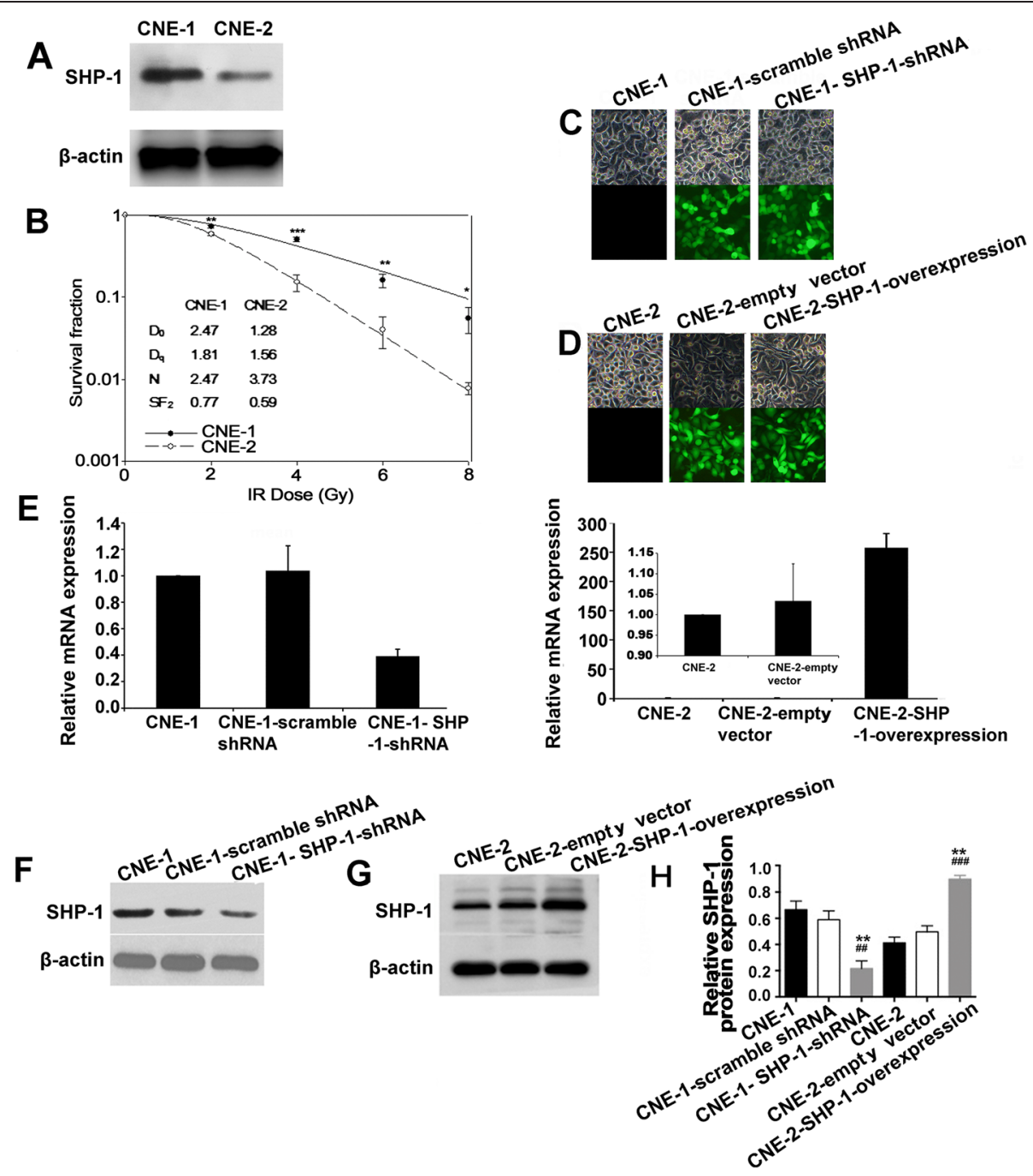

Fig. 1 Alteration of SHP-1 expression in human nasopharyngeal carcinoma (NPC) cell lines CNE-1 and CNE-2 by lentivirus-mediated RNA interference and overexpression, respectively. a SHP-1 protein expression in CNE-1 and CNE-2 cells was determined by western blot. b CNE-1 and CNE-2 cell survival according to radiation dose determined by colony formation assay. ${ }^{*} P<0.05$, ${ }^{* *} P<0.01$, ${ }^{* * *} P<0.001$ CNE-1 vs. CNE-2. CNE-1: non-transduced CNE-1 cells; CNE-1-scramble shRNA: CNE-1 cells transduced with lentivirus-mediated scramble shRNA; CNE-1 SHP-1 shRNA: CNE-1 cells transduced with lentivirus-mediated SHP-1 shRNA; CNE-2: without transduced CNE-2 cells; CNE-2-empty vector: CNE-2 cells transduced with lentivirus vector; CNE-2 SHP-1 overexpression: CNE-2 cells transduced with lentivirus-mediated SHP-1 overexpression. According to fluorescence microscopy, transduction efficiency in CNE-1 (c) and CNE-2 (d) cells was $>90 \%$ at 2 days after transduction (magnification: $\times 400$ ). e SHP-1 mRNA expression were determined by real-time RT-PCR. Relative mRNA expression was normalized to CNE-1 or CNE-2, and $\beta$-actin was used as an inner control. SHP-1 protein expression in CNE-1 (f) and CNE-2 (g) cells was determined by western blot. $\beta$-actin was used as control. $\mathbf{h}$ Quantitative results of western blot are shown as mean \pm standard deviation (SD) from three independent experiments. ${ }^{* * *} P<0.01,{ }^{* * *} P<0.001$ vs. CNE-1 or CNE-2; ${ }^{\# \#} P<0.01,{ }^{\# \# \#} P<0.001$ vs. CNE-1-scramble shRNA or CNE-2-empty vector

$2.7 \%, P<0.001)$, and lower proportions of cells in $\mathrm{S}(14.0$ $\pm 1.7 \%$ vs. $25.0 \pm 3.6 \%, P<0.001)$ and $\mathrm{G} 2 / \mathrm{M}(6.0 \pm 2.0 \%$ vs. $14.8 \pm 4.5 \%, P=0.004)$ phases. Compared with CNE-2empty vector cells, CNE-2 SHP-1 overexpression cells had lower proportions of cells in G1 $(55.7 \pm 2.6 \%$ vs. $71.8 \pm$ $2.9 \%, P<0.001)$ and $\mathrm{G} 2 / \mathrm{M}(4.7 \pm 0.8 \%$ vs. $8.18 \pm 1.3 \%$, $P<0.001)$ phases, and a higher proportion of cells in $\mathrm{S}$ phase $(39.7 \pm 2.2 \%$ vs. $20.1 \pm 2.9 \%, P=0.001)$.

The BrdU assay was used to monitor S-phase progression. Results showed that fewer cells were in the S phase in CNE-1 SHP-1 shRNA cells compared with CNE-1scramble shRNA cells $(21.6 \pm 4.7$ vs. $67.8 \pm 8.4$ cells, $P<$ 0.001 ), while more cells were in the $S$ phase in CNE-2 SHP-1 overexpression cells compared with CNE-2empty vector cells $(88.85 \pm 5.6$ vs. $38.6 \pm 4.0$ cells, $P<$ 0.001) (Fig. 4b).

Compared with CNE-1-scramble shRNA cells, CNE-1 SHP-1 shRNA cells showed decreased expressions of CDK4 $(-44 \%, P<0.001)$, cyclin D1 $(-41 \%, P=0.001)$ and cyclin E $(-97 \%, P<0.001)$. On the other hand, 


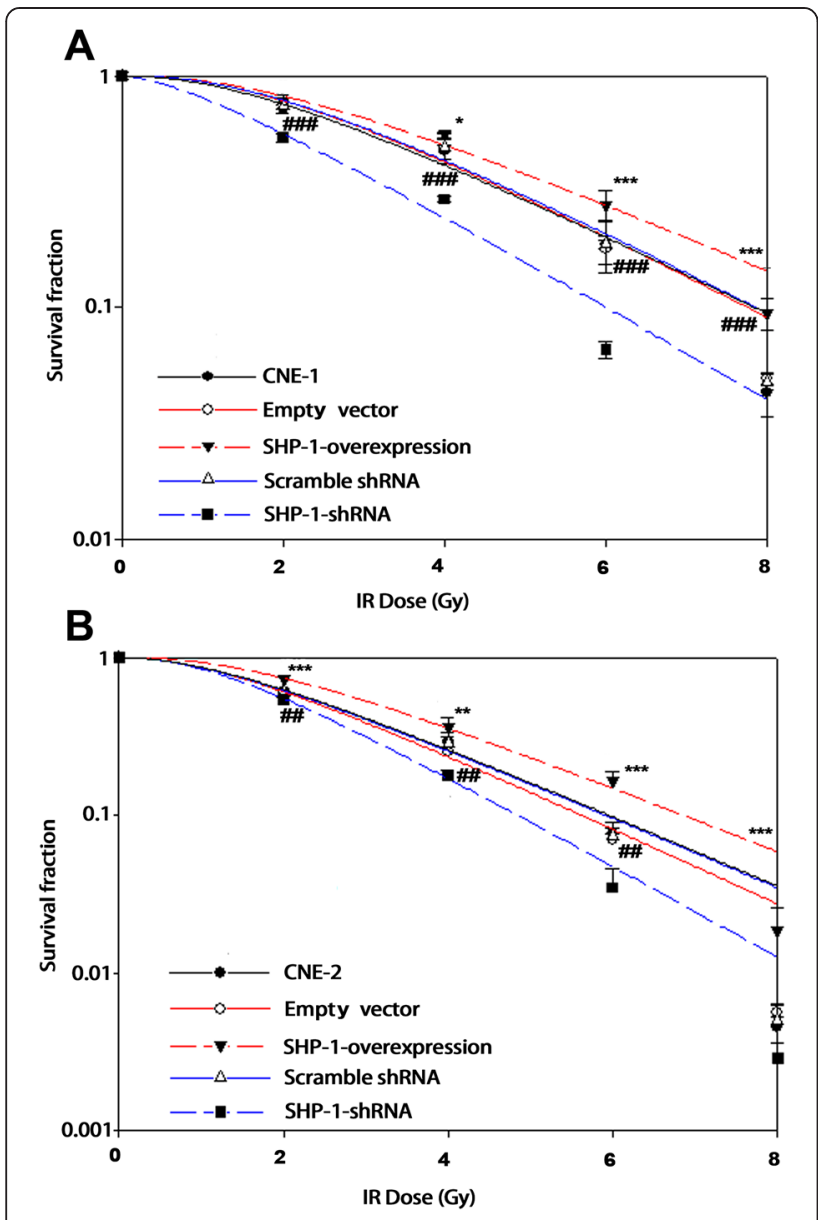

Fig. 2 Effects of SHP-1 knockdown and overexpression on radiosensitivity in $\mathrm{CNE}-1$ and $\mathrm{CNE}-2$ cells. After stable transduction with lentivirus-mediated RNA interference and overexpression, CNE-1 (a) and CNE-2 (b) cells were irradiated at different doses $(0,2,4,6$ and $8 \mathrm{~Gy})$ (radiation absorption rate was $2 \mathrm{~Gy} / \mathrm{min}$ ). After further incubation for 14 days, colony formation assays were used to examine the radiosensitivity of the NPC cells. Survival curves were fitted according to the multi-target single-hit model. Compared with CNE-1 cells, cell survival was significantly different (all $P<0.05$, ANOVA) for all radiation doses in CNE-1 cells with SHP-1 overexpression or silencing. Compared with CNE-2 cells, cell survival was significantly different (all $P<0.05$, ANOVA) for all radiation doses in CNE-2 cells with SHP-1 overexpression or silencing. ${ }^{*} P<0.05,{ }^{* *} P<0.01,{ }^{* * *} P<0.001$ empty vector vs. SHP-1-overexpression; \#\#P<0.01, \#\#\#P<0.001 scramble shRNA vs. SHP-1-shRNA

compared with CNE-2-empty vector cells, CNE-2 SHP-1 overexpression cells showed increased expression of CDK4 $(+41 \%, P<0.001)$, cyclin D1 $(+90 \%, P=0.001)$, and cyclin $\mathrm{E}(+124 \%, P<0.001)$ (Fig. 4c).

\section{Effects of SHP-1 knockdown in CNE-1 cells and overexpression in CNE-2 cells on p16/pRb pathway in NPC cells}

Compared with CNE-1-scramble shRNA cells, CNE-1 SHP-1 shRNA cells showed increased expression of p16
$(+120 \%, P=0.02)$, and decreased expressions of $\mathrm{Rb}$ $(-79 \%, P<0.001)$ and $\mathrm{pRb}(-76 \%, P=0.001)$. On the other hand, compared with CNE-2-empty vector cells, CNE-2 SHP-1 overexpression cells showed decreased expression of p16 $(-95 \%, P<0.001)$, and increased expressions of $\mathrm{Rb}(+358 \%, P<0.001)$ and $\mathrm{pRb}(+248 \%, P<$ 0.001 ) (Fig. 5). Levels of p53 and p21 were unchanged in both cell lines (all $P>0.05$ ).

\section{Discussion}

The aim of the present study was to assess the role of SHP-1 in the radioresistance and senescence of NPC cell lines. Results showed that SHP-1 downregulation resulted in increased senescence, increased radiosensitivity, higher proportion of cells in G0/G1, decreased expression of CDK4, cyclin D1, cyclin E, Rb, and pRb, and increased expression of $\mathrm{p} 16$. On the other hand, overexpression of SHP-1 resulted in decreased senescence, decreased radiosensitivity, higher proportion of cells in S-phase, increased expression of CDK4, cyclin D1, cyclin E, Rb, and pRb, and decreased expression of $\mathrm{p} 16$.

SHP-1 has recently emerged as a useful diagnostic marker and a potential target for therapeutic intervention in several malignancies because of its functional involvement in controlling cell proliferation and tumor cell cycle distribution [29]. Several studies have reported aberrant expression of SHP-1 in different cancers including NPC $[16,15,23,14]$, but no functional study has yet been reported in NPC. SHP-1 overexpression has been reported in NPC and associated with a worse prognosis [23]. Results of the present study showed that SHP-1 is involved in the regulation of the cell cycle and cellular senescence in NPC cells. In addition, SHP-1 levels were higher at baseline in the CNE-1 cells compared with the CNE- 2 cells, and the CNE-1 cells showed higher radioresistance. This is supported by previous studies showing that the CNE-2 cell line has been shown to be less radioresistant than CNE-1 [27], and that the DNA repair mechanisms seem to be more efficient in the CNE-1 cell line $[27,28]$. The results of the present study suggest that SHP-1 might play a role in radioresistance.

A previous study demonstrated that SHP-1 downregulates p16 expression, which in turn interacts and stabilizes FGFR1, thereby promoting epithelial-to-mesenchymal transition and therefore aggressive metastatic behavior of NPC cells [23]. Cellular senescence is an important mechanism for preventing the proliferation of potential cancer cells $[18,19]$. Cellular senescence critically depends on two powerful tumor suppressor pathways: the p53 and $\mathrm{pRb} / \mathrm{p} 16 \mathrm{INK}$ 4a pathways $[22,21,20]$. Both pathways integrate multiple aspects of cellular physiology to determine and orchestrate cell fate. Senescence growth arrest usually depends on the activation of the CDK inhibitors (p21CIP1 and p16INK4A) [30,31], components of the tumor- 
Table 1 Effects of SHP-1 knockdown and overexpression in CNE-1 and CNE-2 cells on radiosensitivity parameters

\begin{tabular}{|c|c|c|c|c|c|c|c|c|c|c|}
\hline $\begin{array}{l}\text { Radiosensitivity } \\
\text { parameters }\end{array}$ & CNE-1 & $\begin{array}{l}\text { CNE-1- } \\
\text { empty } \\
\text { vector }\end{array}$ & $\begin{array}{l}\text { CNE-1- } \\
\text { scramble } \\
\text { shRNA }\end{array}$ & $\begin{array}{l}\text { CNE-1- SHP-1- } \\
\text { overexpression }\end{array}$ & $\begin{array}{l}\text { CNE-1- } \\
\text { SHP-1- } \\
\text { ShRNA }\end{array}$ & CNE-2 & $\begin{array}{l}\text { CNE-2- } \\
\text { empty vector }\end{array}$ & $\begin{array}{l}\text { CNE-2- } \\
\text { scramble shRNA }\end{array}$ & $\begin{array}{l}\text { CNE-2- SHP-1- } \\
\text { overexpression }\end{array}$ & $\begin{array}{l}\text { CNE-2- } \\
\text { SHP-1- shRNA }\end{array}$ \\
\hline SF2 & 0.75 & 0.78 & 0.78 & 0.82 & 0.56 & 0.62 & 0.60 & 0.61 & 0.74 & 0.55 \\
\hline$D_{0}$ & 2.51 & 2.38 & 2.38 & 2.85 & 2.17 & 1.94 & 1.80 & 1.94 & 2.08 & 1.50 \\
\hline$D_{q}$ & 1.77 & 1.85 & 1.85 & 1.96 & 1.26 & 1.46 & 1.43 & 1.43 & 1.75 & 1.36 \\
\hline N & 2.34 & 2.68 & 2.65 & 2.48 & 1.62 & 2.22 & 2.32 & 2.17 & 2.77 & 2.58 \\
\hline
\end{tabular}

Note: SF2: cell survival fraction with 2 cGy irradiation dose; D0: mean lethal dose value; Dq: quasithreshold dose; N: extrapolation number

suppressor pathways governed by $\mathrm{p} 53$ and $\mathrm{pRb}$, and is generally associated with a G0/G1 cell cycle arrest. SAHF formation coincides with the recruitment of heterochromatin proteins and $\mathrm{Rb}$ to E2F-responsive promoters and is associated with the stable repression of E2F target genes [32]. Notably, both SAHF formation and the silencing of
E2F target genes depend on the integrity of the Rb pathway and do not occur in reversibly arrested cells [33]. Often lost in a variety of malignancies, p16 acts as an allosteric inhibitor of the CDK4/6 complex to prevent its interaction with cyclin D1, inducing cell cycle arrest and senescence by activating the Rb pathway [34]. The CDK4/

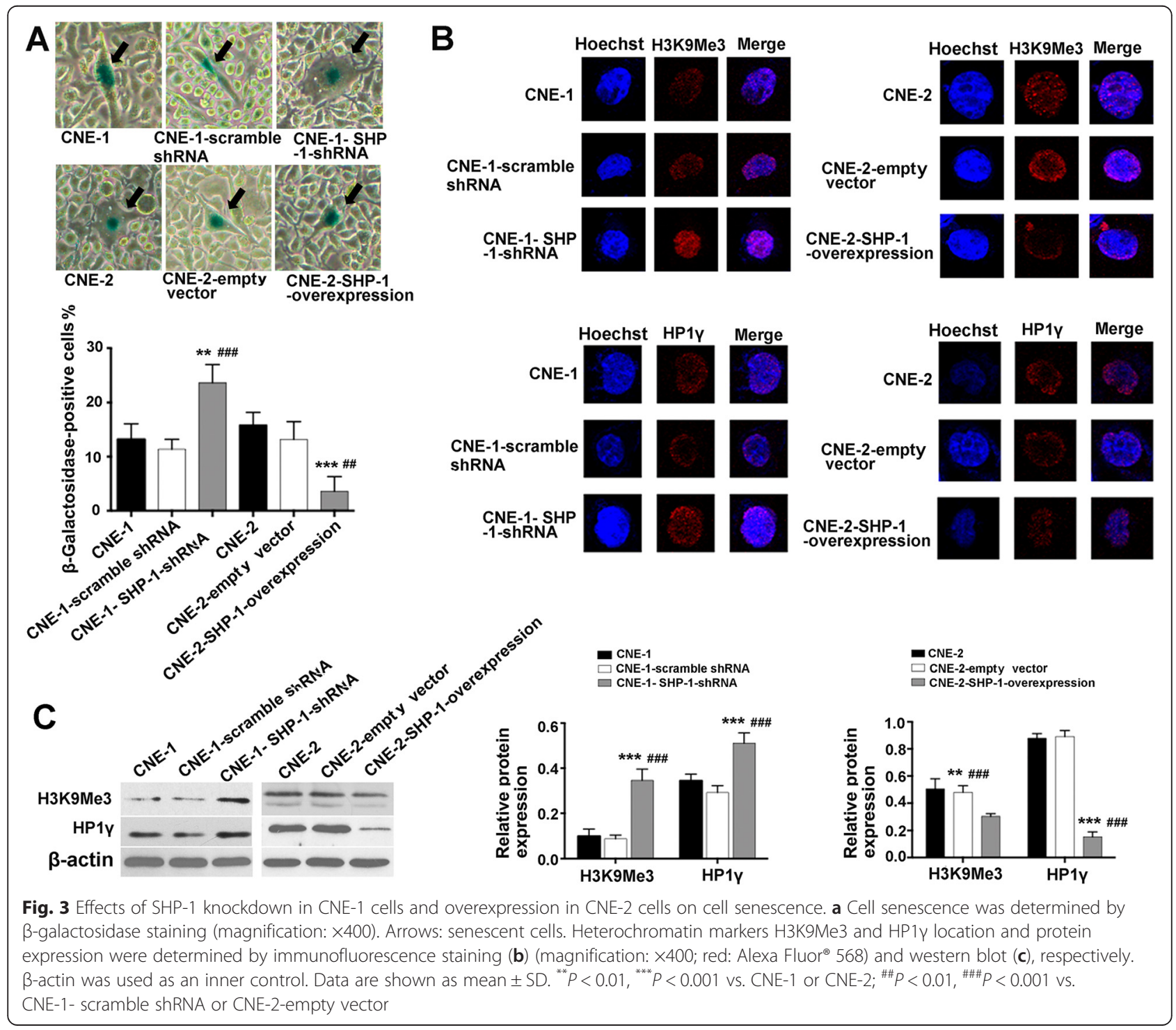




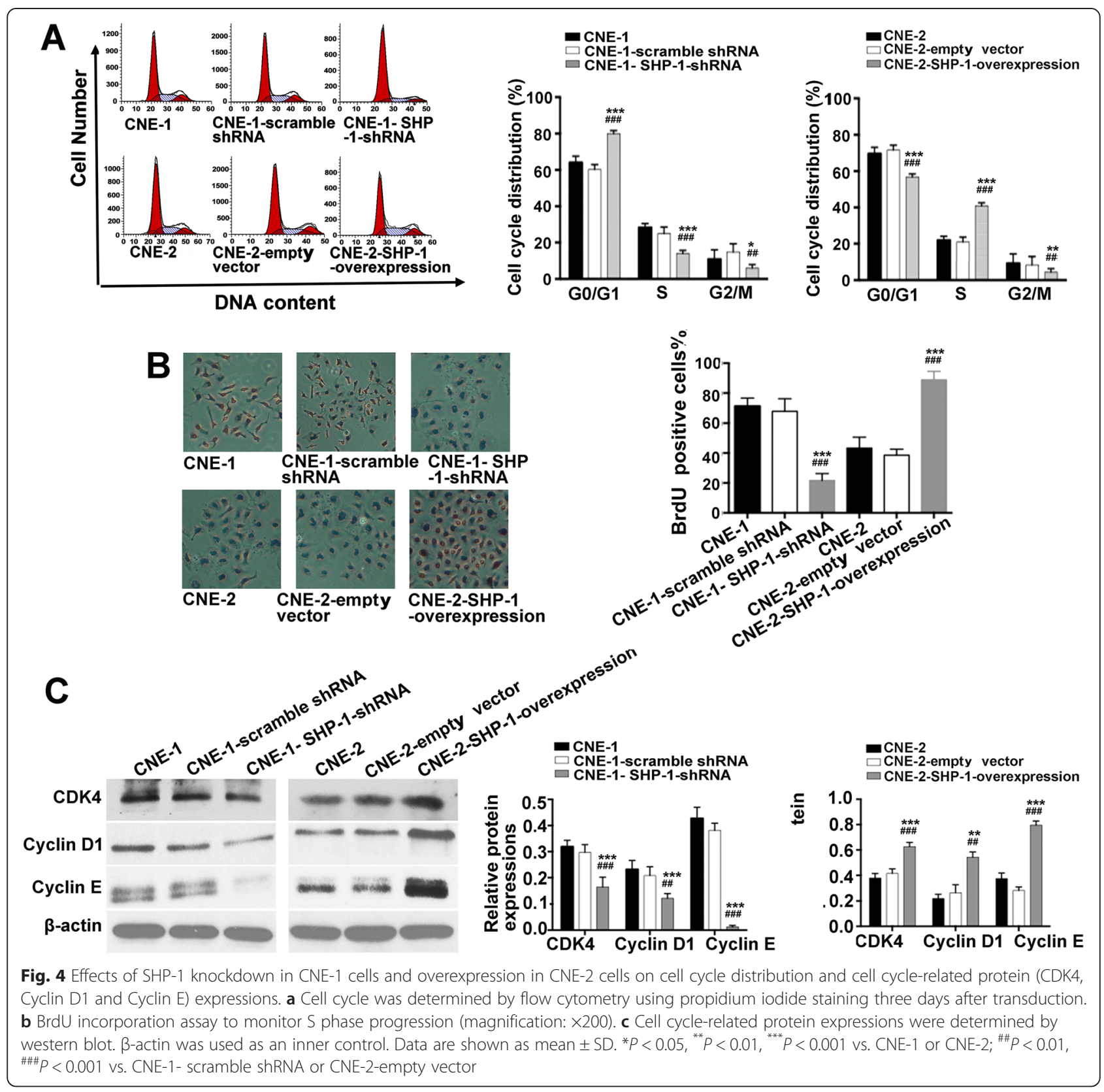

6-cyclin D1 complex-mediated phosphorylation and inactivation of $\mathrm{Rb}$ allows the transcription of E2F-dependent various cell cycle regulatory genes including cyclin E [34].

In the present study, stable suppression of SHP-1 mRNA in CNE-1 cells resulted in increased radiosensitivity compared with the parental cells, a decrease in the number of cells in S-phase and an increase in the expression of p16. Furthermore, we observed that SHP-1 increased cell proliferation by modulating cell cycle regulatory proteins like p16, CDK4 and cyclin D1. SHP1 silencing suppressed growth via a unique mechanism involving G0/G1 cell cycle arrest. Interestingly, SHP-1 silencing in NPC cells resulted in cellular senescence, as suggested by cell morphology, increased SA- $\beta$-Gal-stained cells, and SAHF formation, which are considered to be characteristics of senescent cells [35]. Although the involvement of p16 in cellular senescence and its downregulation in NPC is well established [23], there is still a lack of comprehensive studies about its role in NPC senescence. Increased proliferation is mostly driven by altered cell cycle progression. In the present study, downregulation of SHP-1 resulted in decreased proliferation 


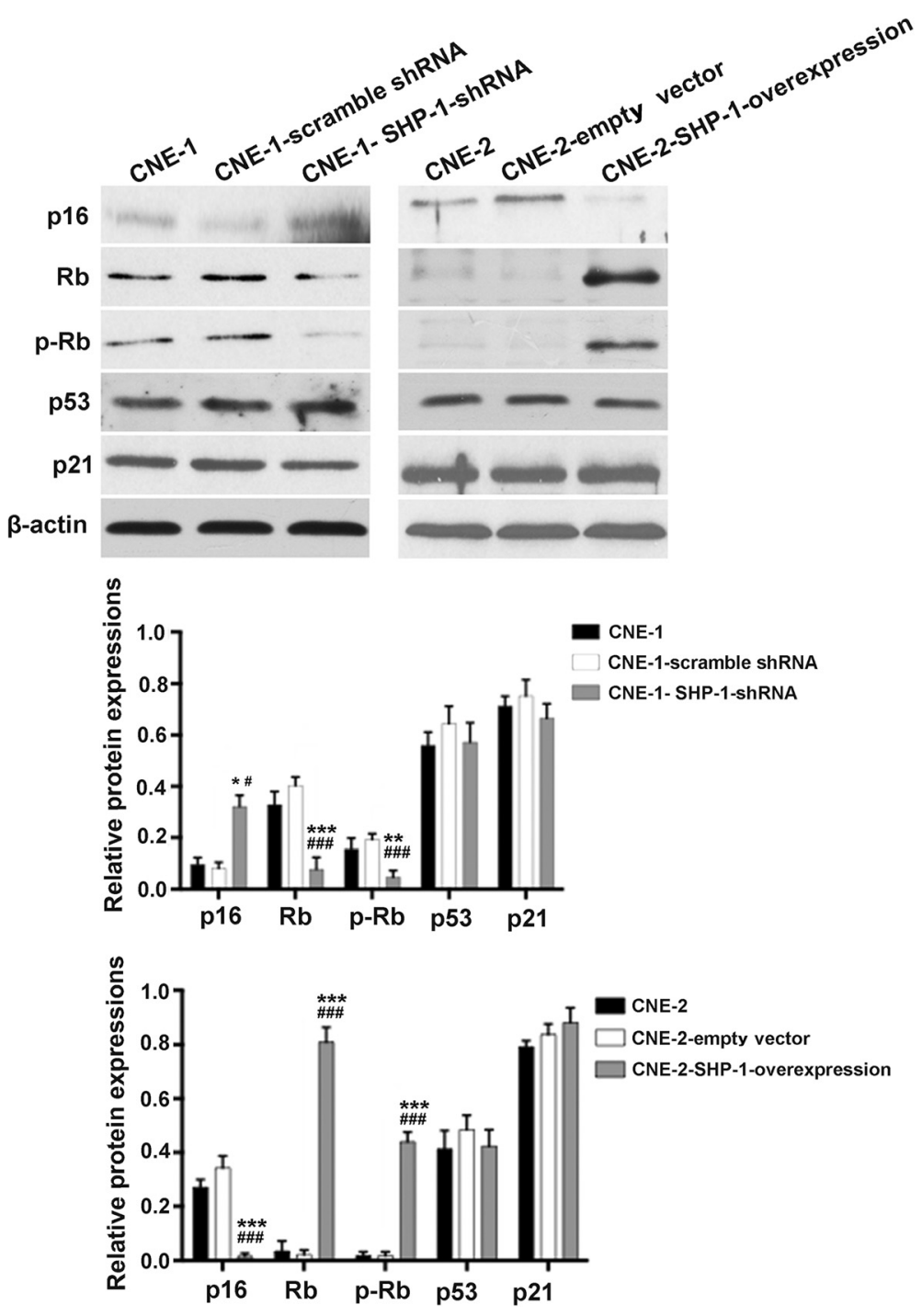

Fig. 5 Effects of SHP-1 knockdown in CNE-1 cells and overexpression in CNE-2 cells on senescence and cell cycle-related signaling molecules ( $\mathrm{p} 16, \mathrm{Rb}, \mathrm{p}-\mathrm{Rb}, \mathrm{p} 53, \mathrm{p} 21)$ expression. Protein expressions were determined by western blot. $\beta$-actin was used as control. Data are shown as mean \pm SD. ${ }^{*} P<0.05,{ }^{* *} P<0.01,{ }^{* * * *} P<0.001$ vs. CNE-1 or CNE-2; ${ }^{\#} P<0.05,{ }^{\# \# \#} P<0.001$ vs. CNE-1- scramble shRNA or CNE-2-empty vector

and G0/G1 cell cycle arrest, providing evidence that SHP-1 is a modulator of the cell cycle. Decreased proliferation and cell cycle arrest was associated with downregulation of cell cycle regulatory proteins such as CDK4 and cyclin D1. However, modulation of SHP-1 expression had no effect on expression levels of p53 and p21, two proteins that play important roles in cell cycle progression [36]. Cyclin E/D downregulation might be a direct consequence of SHP-1 depletion, but further study is necessary to elucidate this issue.

The present study is not without limitations. Indeed, we could not investigate the PTEN/p27KIP1 pathway in the present study. In addition, only cell lines were used, and the observation of SHP-1 in actual tumors should be beneficial. However, we plan to study this association in the near future.

\section{Conclusions}

In conclusion, we observed that SHP-1 downregulation or overexpression affected radioresistance, cell senescence and cell cycle distribution in NPC cell lines. These findings not only offer new perspectives in the modulation of senescence by SHP-1, but also provide strong evidence for SHP-1-based therapeutic interventions in NPC patients.

\section{Abbreviations}

ANOVA: Analysis of variance; HP1 $ү$ : Heterochromatin protein-1 $\gamma$; NPC: Nasopharyngeal carcinoma; RT: Radiotherapy; SD: Standard deviation; SNK: Student-Newman-Keuls. 


\section{Competing interests}

The authors declare that they have no competing interests.

\section{Authors' contributions}

ZYS and XFP carried out the study design, data collection and analysis, wrote the manuscript. ZWZ, QD and GW participated in data collection, statistical analysis, and provided the critical revision. GP conceived of the study, and participated in its design and coordination and provided the critical revision. All authors read and approved the final manuscript.

\section{Authors' information}

Ziyi Sun and Xiaofen Pan are co-first author.

\section{Acknowledgements}

This work was supported by grants from the Natural Sciences Foundation of China (No. 81301976) and the Wu Jieping Medical Foundation.

\section{Author details}

${ }^{1}$ Cancer Center, Union hosipital, Wuhan 430022, Hubei Province, China. ${ }^{2}$ Cancer center, Affliated Hospital of Guangdong Medical College, Zhanjiang 524001, Guangdong Province, China.

Received: 2 March 2015 Accepted: 23 June 2015

Published online: 28 July 2015

\section{References}

1. Wei WI, Sham JS. Nasopharyngeal carcinoma. Lancet. 2005:365:2041-54.

2. Anantharaman D, Marron M, Lagiou P, Samoli E, Ahrens W, Pohlabeln H, et al. Population attributable risk of tobacco and alcohol for upper aerodigestive tract cancer. Oral Oncol. 2011;47:725-31.

3. Freedman ND, Abnet CC, Leitzmann MF, Hollenbeck AR, Schatzkin A. Prospective investigation of the cigarette smoking-head and neck cancer association by sex. Cancer. 2007;110:1593-601.

4. Chan AT, Gregoire V, Lefebvre JL, Licitra L, Hui EP, Leung SF, et al. Nasopharyngeal cancer: EHNS-ESMO-ESTRO Clinical Practice Guidelines for diagnosis, treatment and follow-up. Ann Oncol. 2012;23 Suppl 7:vii83-5.

5. Chien YC, Chen JY, Liu MY, Yang HI, Hsu MM, Chen CJ, et al. Serologic markers of Epstein-Barr virus infection and nasopharyngeal carcinoma in Taiwanese men. N Engl J Med. 2001;345:1877-82.

6. Mork J, Lie AK, Glattre E, Hallmans G, Jellum E, Koskela P, et al. Human papillomavirus infection as a risk factor for squamous-cell carcinoma of the head and neck. N Engl J Med. 2001;344:1125-31.

7. Medow MA, Weed HG, Schuller DE. Simple predictors of survival in head and neck squamous cell carcinoma. Arch Otolaryngol Head Neck Surg. 2002;128:1282-6.

8. Fang FM, Chien CY, Tsai WL, Chen HC, Hsu HC, Lui CC, et al. Quality of life and survival outcome for patients with nasopharyngeal carcinoma receiving three-dimensional conformal radiotherapy vs. intensity-modulated radiotherapy-a longitudinal study. Int J Radiat Oncol Biol Phys. 2008;72:356-64

9. Yeh SA, Tang Y, Lui CC, Huang YJ, Huang EY. Treatment outcomes and late complications of 849 patients with nasopharyngeal carcinoma treated with radiotherapy alone. Int J Radiat Oncol Biol Phys. 2005:62:672-9.

10. Feng XP, Yi H, Li MY, Li XH, Yi B, Zhang PF, et al. Identification of biomarkers for predicting nasopharyngeal carcinoma response to radiotherapy by proteomics. Cancer Res. 2010;70:3450-62.

11. Chang JT, Chan SH, Lin CY, Lin TY, Wang HM, Liao CT, et al. Differentially expressed genes in radioresistant nasopharyngeal cancer cells: gp96 and GDF15. Mol Cancer Ther. 2007:6:2271-9.

12. Qu C, Liang Z, Huang J, Zhao R, Su C, Wang S, et al. MiR-205 determines the radioresistance of human nasopharyngeal carcinoma by directly targeting PTEN. Cell Cycle. 2012;11:785-96.

13. Lorenz U. SHP-1 and SHP-2 in T cells: two phosphatases functioning at many levels. Immunol Rev. 2009;228:342-59.

14. Wu C, Sun M, Liu L, Zhou GW. The function of the protein tyrosine phosphatase SHP-1 in cancer. Gene. 2003;306:1-12.

15. Evren S, Wan S, Ma XZ, Fahim S, Mody N, Sakac D, et al. Characterization of SHP-1 protein tyrosine phosphatase transcripts, protein isoforms and phosphatase activity in epithelial cancer cells. Genomics. 2013;102:491-9.

16. Amin S, Kumar A, Nilchi L, Wright K, Kozlowski M. Breast cancer cells proliferation is regulated by tyrosine phosphatase SHP1 through c-jun
N-terminal kinase and cooperative induction of RFX-1 and AP-4 transcription factors. Mol Cancer Res. 2011:9:1112-25.

17. Sharpless NE, DePinho RA. Cancer: crime and punishment. Nature. 2005;436:636-7.

18. Cairney CJ, Bilsland AE, Evans TR, Roffey J, Bennett DC, Narita M, et al. Cancer cell senescence: a new frontier in drug development. Drug Discov Today. 2012;17:269-76

19. Campisi J. Aging, cellular senescence, and cancer. Annu Rev Physiol. 2013;75:685-705

20. Reinhardt HC, Schumacher B. The p53 network: cellular and systemic DNA damage responses in aging and cancer. Trends Genet. 2012;28:128-36.

21. Purvis JE, Karhohs KW, Mock C, Batchelor E, Loewer A, Lahav G. p53 dynamics control cell fate. Science. 2012:336:1440-4.

22. Bian Y, Hall B, Sun ZJ, Molinolo A, Chen W, Gutkind JS, et al. Loss of TGFbeta signaling and PTEN promotes head and neck squamous cell carcinoma through cellular senescence evasion and cancer-related inflammation. Oncogene. 2012;31:3322-32.

23. Peng G, Cao RB, Li YH, Zou ZW, Huang J, Ding Q. Alterations of cell cycle control proteins SHP1/2, p16, CDK4 and cyclin D1 in radioresistant nasopharyngeal carcinoma cells. Mol Med Rep. 2014;10:1709-16.

24. Hwang CF, Cho CL, Huang CC, Wang JS, Shih YL, Su CY, et al. Loss of cyclin D1 and p16 expression correlates with local recurrence in nasopharyngeal carcinoma following radiotherapy. Ann Oncol. 2002;13:1246-51.

25. Zeng Y. Establishment of an epitheloid cell line and a fusiform cell line from a patient with nasopharyngeal carcinoma. Sci Sin. 1978;21:127-34.

26. Gu S, Tang W, Zeng Y, Zhao M. An epithelial cell line established from poorly differentiated nasopharyngeal carcinoma. Chin J Cancer. 1983;2:70-6.

27. He YX, Zhong PP, Yan SS, Liu L, Shi HL, Zeng MS, et al. DNA-dependent protein kinase activity and radiosensitivity of nasopharyngeal carcinoma cell lines CNE1/CNE2. Sheng Li Xue Bao. 2007;59:524-33.

28. Wang HM, Wu XY, Xia YF, Qian JY. Expression of ATM protein in nasopharyngeal carcinoma cell lines with different radiosensitivity. Ai Zheng. 2003;22:579-81.

29. Fan LC, Teng HW, Shiau CW, Lin H, Hung MH, Chen YL, et al. SHP-1 is a target of regorafenib in colorectal cancer. Oncotarget. 2014;5:6243-51.

30. Li XN, Shu Q, Su JM, Perlaky L, Blaney SM, Lau CC. Valproic acid induces growth arrest, apoptosis, and senescence in medulloblastomas by increasing histone hyperacetylation and regulating expression of p21Cip1, CDK4, and CMYC. Mol Cancer Ther. 2005;4:1912-22.

31. Collado M, Gil J, Efeyan A, Guerra C, Schuhmacher AJ, Barradas M, et al. Tumour biology: senescence in premalignant tumours. Nature. 2005;436:642.

32. Maehara K, Yamakoshi K, Ohtani N, Kubo Y, Takahashi A, Arase S, et al. Reduction of total E2F/DP activity induces senescence-like cell cycle arrest in cancer cells lacking functional pRB and p53. J Cell Biol. 2005;168:553-60.

33. Narita M, Narita M, Krizhanovsky V, Nunez S, Chicas A, Hearn SA, et al. A novel role for high-mobility group a proteins in cellular senescence and heterochromatin formation. Cell. 2006;126:503-14

34. Ely S, Di Liberto M, Niesvizky R, Baughn LB, Cho HJ, Hatada EN, et al. Mutually exclusive cyclin-dependent kinase 4/cyclin D1 and cyclindependent kinase 6/cyclin D2 pairing inactivates retinoblastoma protein and promotes cell cycle dysregulation in multiple myeloma. Cancer Res. 2005;65:11345-53.

35. Cho KA, Ryu SJ, Oh YS, Park JH, Lee JW, Kim HP, et al. Morphological adjustment of senescent cells by modulating caveolin-1 status. J Biol Chem. 2004;279:42270-8.

36. Narayanan BA, Geoffroy O, Willingham MC, Re GG, Nixon DW. p53/ p21(WAF1/CIP1) expression and its possible role in G1 arrest and apoptosis in ellagic acid treated cancer cells. Cancer Lett. 1999;136:215-21. 